\title{
The Impact of Age on Clinical Outcomes of Coronary Artery Bypass Grafting: Long-Term Results of a Real-World Registry
}

\author{
Francesco Nicolini, ${ }^{1}$ Daniela Fortuna, ${ }^{2}$ Giovanni Andrea Contini, ${ }^{3}$ \\ Davide Pacini, ${ }^{4}$ Davide Gabbieri, ${ }^{5}$ Claudio Zussa, ${ }^{6}$ Rossana De Palma, \\ Antonella Vezzani, ${ }^{3}$ and Tiziano Gherli ${ }^{1}$ \\ ${ }^{1}$ Cardiac Surgery Unit, Department of Medicine and Surgery, University of Parma, Parma, Italy \\ ${ }^{2}$ Regional Agency for Health and Social Care, Emilia-Romagna, Italy \\ ${ }^{3}$ Cardiac Surgery Unit, Surgery Department, Parma Hospital, Parma, Italy \\ ${ }^{4}$ Cardio-Thoracic-Vascular Department, University Hospital S. Orsola-Malpighi, Bologna, Italy \\ ${ }^{5}$ Department of Clinical Cardiology and Thoraco Vascular Surgery, Hesperia Hospital, Modena, Italy \\ ${ }^{6}$ Department of Cardiology and Cardiac Surgery, Villa Maria Cecilia Hospital, Lugo, Ravenna, Italy \\ Correspondence should be addressed to Francesco Nicolini; francesco.nicolini@unipr.it
}

Received 27 May 2017; Revised 26 September 2017; Accepted 19 October 2017; Published 20 December 2017

Academic Editor: Betti Giusti

Copyright (c) 2017 Francesco Nicolini et al. This is an open access article distributed under the Creative Commons Attribution License, which permits unrestricted use, distribution, and reproduction in any medium, provided the original work is properly cited.

\begin{abstract}
The aim of this retrospective multicenter registry study was to investigate age-dependent trends in mortality, long-term survival, and comorbidity over time in patients who underwent isolated CABG from 2003 to 2015 . The percentage of patients $<60$ years of age was $18.9 \%$. Female sex, chronic pulmonary disease, extracardiac arteriopathy, and neurologic dysfunction disease were significantly less frequent in this younger population. The prevalence of $\mathrm{BMI} \geq 30$, previous myocardial infarction, preoperative severe depressed left ventricular ejection fraction, and history of previous PCI were significantly higher in this population. After PS matching, at 5 years, patients $<60$ years of age reported significantly lower overall mortality $(p<0.0001)$, cardiac-related mortality $(p<$ $0.0001)$, incidence of acute myocardial infarction $(p=0.01)$, and stroke rates $(p<0.0001)$. Patients $<60$ years required repeated revascularization more frequently than older patients $(p=0.05)$. Patients $<60$ who underwent CABG had a lower risk of adverse outcomes than older patients. Patients $<60$ have a different clinical pattern of presentation of CAD in comparison with more elderly patients. These issues require focused attention in order to design and improve preventive strategies aiming to reduce the impact of specific cardiovascular risk factors for younger patients, such as diet, lifestyle, and weight control.
\end{abstract}

\section{Introduction}

Premature coronary artery disease in young patients (CAD) is a rapidly progressive form of the disease [1], but it requires invasive revascularization infrequently $[2,3]$.

Numerous studies reported that young patients with CAD have a significant prevalence of classic cardiovascular risk factors $[4,5]$, and that the premature clinical onset of their symptoms can be more aggressive than in elderly patients [6]. In fact, young adults who undergo coronary artery revascularization are a specific subpopulation of patients, and to date there have been few studies on survival data, cardiovascular events, or the need for repeated revascularization $[3,7]$.
It is clear that the results of revascularization in young patients need to be durable, in order to avoid recurrence of symptoms or cardiovascular events and the need for repeat revascularization. Young patients undergoing coronary artery bypass grafting $(\mathrm{CABG})$ demonstrate survival rates similar to those of percutaneous coronary intervention (PCI), but lower rates of repeated revascularization [7]. The majority of recent studies of CABG outcomes investigate only the risks for elderly patients undergoing coronary revascularization [8-12], but there are few long-term reports of the impact of age stratification on CABG outcomes, particularly for young patients.

The aim of this retrospective multicenter registry study was to investigate age-dependent trends in mortality, long-term 
survival, and comorbidity over time, in a population of patients who underwent isolated CABG between 2003 and 2015.

\section{Materials and Methods}

2.1. Data Source. Emilia-Romagna (ER) is an Italian region with about 4 million inhabitants. Cardiac surgery is performed by six hospitals (two public university hospitals and four private hospitals). The RERIC Registry (Registro dell'Emilia Romagna degli Interventi Cardiochirurgici) is a prospective regional database designed in 2002 by the Agency for Health and Social Care of ER region, with the aim of collecting pre-, intra-, and postoperative data from all the patients undergoing cardiac surgical procedures in the region. The rationale and methodology of RERIC have been published previously $[12,13]$. The Regional Agency for Health and Social Care ensures data quality/completeness control. The RERIC registry is linked to the ER regional mortality registry and to the regional hospital admission database, in order to collect accurate information on the occurrence of follow-up mortality and morbidity. Variables and events occurring after the index hospital discharge have also been collected from outpatient clinics at the individual Institutions. In case of absent/missing data, variables and events have been collected by direct phone contact with general practitioners and only if persistently missed by phone contact with patients and families. This registry is based on current clinical practice. The requirement for individual patient consent was waived because of the retrospective design of the study and because data were collected from routine care procedures. All data were anonymized and deidentified prior to analysis by the central statistical laboratory of the Regional Agency for Health and Social Care. The protocol of the study is in accordance with the Declaration of Helsinki.

2.2. Study Population. From January 1, 2003 to December 31, 2015, data of all patients undergoing CABG were collected in the RERIC Registry. Exclusion study criteria were emergency, cardiogenic shock, associated valve surgery procedures, major aortic surgery, and supra-aortic vessels disease requiring surgery. Patients with a previous cardiac operation and requiring only isolated $\mathrm{CABG}$ at the time of RE-DO surgery were included in the study. After these exclusions, we filtered 10,597 patients subjected to isolated CABG. Additional exclusion criteria were not being resident in ER (administrative follow-up not feasible for 2320 patients) and the presence of incomplete information about baseline and procedural characteristics. The remaining 8277 patients were followed through January 30, 2017. The patients were divided into four age bands for the purposes of the statistical analysis: $<60$ years $(n=1564), 60-69$ years $(n=2913), 70-79$ years $(n=3200)$, and $\geq 80$ years $(n=600)$ (Figure 1$)$.

2.3. Procedures. Decisions about the type of treatment were taken according to local practices and there were no standard regional protocols. The choice of CABG technique, performed either with the use of extracorporeal circulation (ECC) or off-pump, was left to the surgeon's discretion. Whenever possible, the left internal thoracic artery (LIMA) was used preferentially for revascularization of the left anterior descending artery (LAD). Complete revascularization was performed with other arterial conduits, namely, right internal mammary artery (RIMA) and radial artery (RA) or saphenous vein grafts.

Follow-up angiography was not performed routinely in either group of patients.

2.4. Definition of the Outcomes. All-cause death included overall mortality occurring during the index hospital admission or thereafter. Cardiac death was defined as any death due to a cardiac cause (e.g., myocardial infarction (MI), low output failure, and fatal arrhythmia), and other types were procedure-related death and death of unknown cause. Acute MI was defined as any hospital admission occurring after the index procedure with a principal diagnosis of MI. This adverse event is defined according to the recent definition criteria by Moussa et al. [14]. Stroke included complications at the index admission and further hospital admissions with stroke as principal diagnosis. Overall rehospitalization was defined as any hospital admission after the index procedure due to a cardiac cause (e.g., MI, repeat PCI, repeat CABG, new pacemaker implantation, new occurrence of heart failure, or need for long-term hospital care). Repeat PCI was defined as any percutaneous coronary procedure during follow-up, treating a luminal stenosis in the same coronary vessel treated at the index procedure or treating other native vessel stenosis not previously revascularized.

2.5. Statistical Analysis. The study population was classified into 4 different age bands: less than 60 years, 60 to 69 years, 70 to 79 years, and 80 years or older, respectively. Demographic and clinical features of the patients were presented as counts and percentages and were compared between the four age classes, using the Chi-square test or Fisher's exact test when appropriate. For each class of age, cumulative risk curves of assessed outcomes (death for all causes, including perioperative deaths, cardiac death, myocardial infarction, stroke, repeat revascularization with PCI, and repeat hospitalization) were estimated at 5 years using the Kaplan-Meier method and were also compared by log-rank test.

Patients were then split into two groups, using a cut-off age of 60 years. Multivariate logistic regression analysis, with a binary dependent variable representing $<60$ years versus $>60$ years, was performed to estimate propensity score (PS) of treatment. Independent variables included demographics and the available clinical characteristics. Patients were matched on the logit of the PS using a caliper of width equal to 0.25 standard deviations of the logit of PS. Appropriateness of the specification of the PS was assessed by examining the degree to which matching on the estimated PS resulted in a matched sample in which the distribution of measured baseline covariates was similar between the two groups. Imbalances in baseline covariates were detected by standardized differences. Standardized differences of less than $0.10(10 \%)$ are likely to indicate a negligible imbalance between the two groups.

Kaplan-Meier estimates were used to plot the rates of the long-term adverse events, and differences between risk curves 


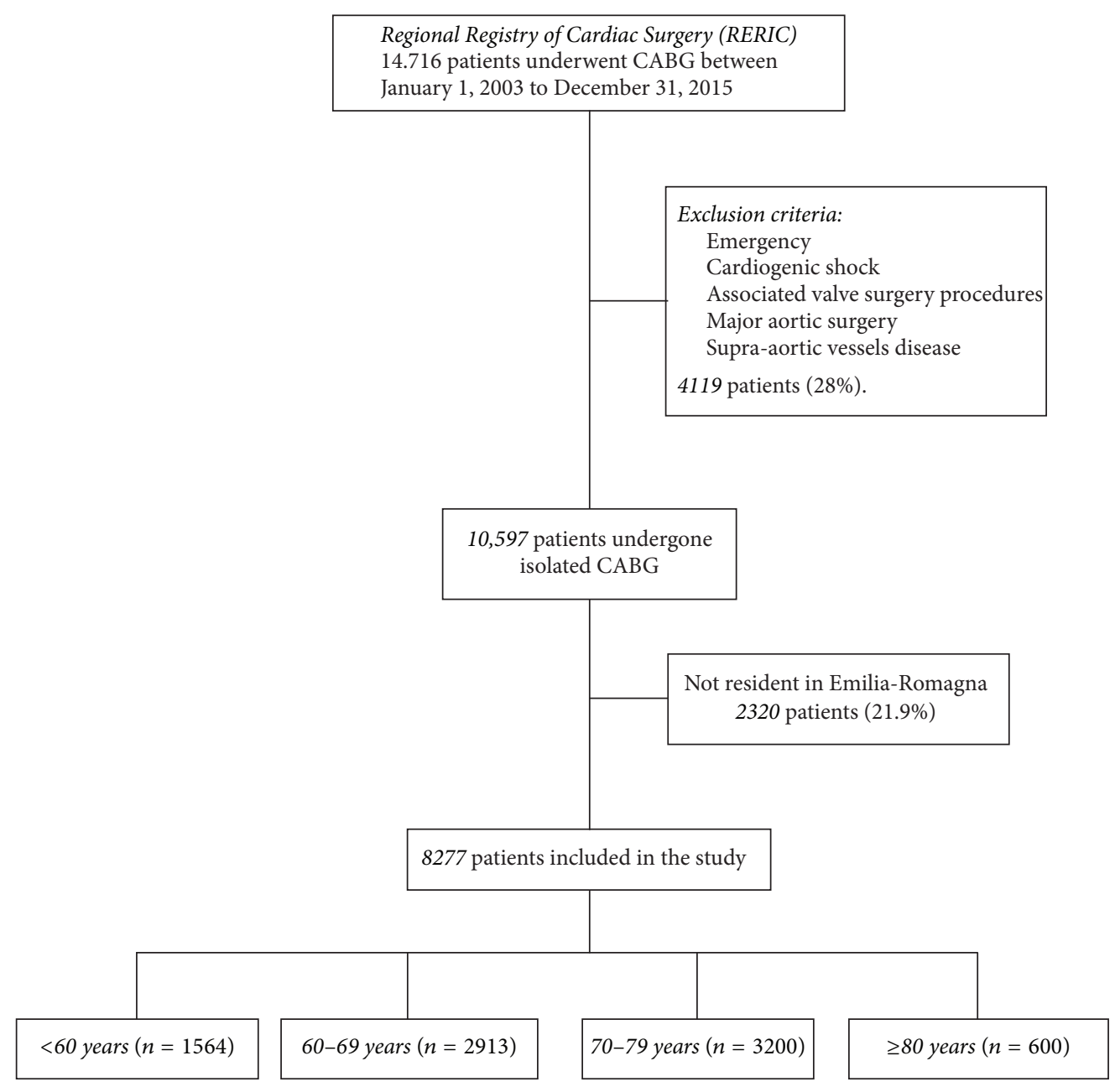

FIgURE 1: Selection criteria.

were assessed using the Klein-Moeschberger test for matched pairs. Independent predictors of 5-year mortality risk were estimated using a stepwise multivariable Cox proportional hazards model, with robust standard errors to account for clustering in matched pairs including treatment and individual covariates including all pre- and intraoperative variables available.

All the analyses were performed with SAS version 9.3.

\section{Results}

A reduction in the number of overall isolated elective CABG was observed in our registry between 2003 and 2015, although a plateau can be seen in the last five years (Figure 2).

The entire study cohort showed that patient risk profiles differed significantly between the groups (Table 1 ). The prevalence of patients under 60 is $18.9 \%$ (1564 of 8277 patients). Patients over 60 show a significantly lower prevalence of baseline comorbidities (Table 1). In particular, female sex, chronic pulmonary disease, extracardiac arteriopathy, and neurologic dysfunction disease were significantly less frequent in this younger population (Table 1). On the other

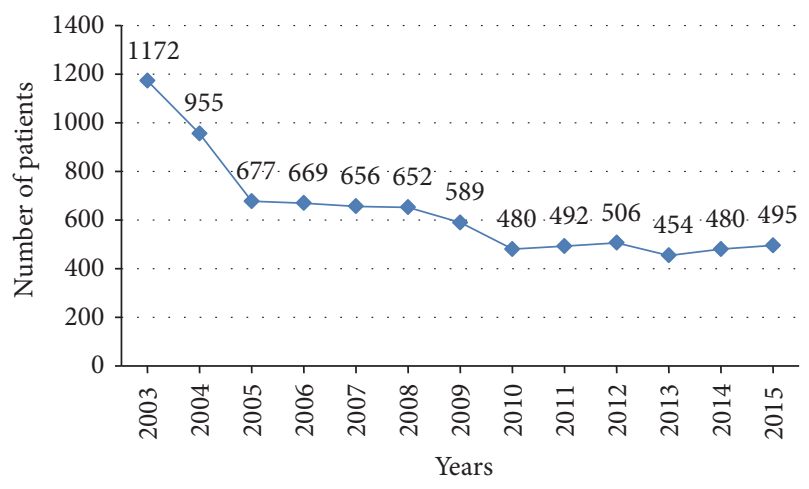

FIGURE 2: CABG trend over years in Emilia-Romagna region (Italy).

hand, the prevalence of $\mathrm{BMI} \geq 30 \mathrm{Kg} / \mathrm{m}^{2}$ was significantly higher in the under 60 group. Previous MI was reported in about one-third of patients $<60$ and was significantly higher than in patients aged 60-69 and 70-79 years. Moreover, patients $<60$ reported more frequently a preoperative severe depressed left ventricular ejection fraction (LVEF), although 
TABLE 1: Baseline characteristics and operative data of patients according to different age classes.

\begin{tabular}{|c|c|c|c|c|c|c|c|c|c|}
\hline \multirow[t]{2}{*}{ Patients' characteristics } & \multicolumn{2}{|c|}{$\begin{array}{c}<60 \text { yrs } \\
(N=1564)\end{array}$} & \multicolumn{2}{|c|}{$\begin{array}{c}60-69 \text { yrs } \\
(N=2913)\end{array}$} & \multicolumn{2}{|c|}{$\begin{array}{c}70-79 \text { yrs } \\
(N=3200)\end{array}$} & \multicolumn{2}{|c|}{$\begin{array}{c}\geq 80 \text { yrs } \\
(N=600)\end{array}$} & \multirow[t]{2}{*}{$p$} \\
\hline & $N$ & $\%$ & $N$ & $\%$ & $N$ & $\%$ & $N$ & $\%$ & \\
\hline Female & 154 & 9.8 & 416 & 14.3 & 726 & 22.7 & 181 & 30.2 & $<0.0001$ \\
\hline $\mathrm{BMI} \geq 30 \mathrm{~kg} / \mathrm{m}^{2}:$ obesity & 402 & 25.7 & 595 & 20.4 & 486 & 15.2 & 58 & 9.7 & $<0.0001$ \\
\hline Logistic EUROscore > 15\% & 3 & 0.2 & 36 & 1.2 & 205 & 6.4 & 136 & 22.7 & $<0.0001$ \\
\hline Critical preoperative state & 21 & 1.3 & 30 & 1.0 & 45 & 1.4 & 10 & 1.7 & 0.457 \\
\hline Unstable angina & 101 & 6.5 & 203 & 7.0 & 243 & 7.6 & 85 & 14.2 & $<0.0001$ \\
\hline $\mathrm{LVEF} \leq 30 \%$ & 44 & 2.8 & 75 & 2.6 & 73 & 2.3 & 13 & 2.2 & 0.661 \\
\hline LVEF $30 \%-60 \%$ & 370 & 23.7 & 697 & 23.9 & 869 & 27.2 & 209 & 34.8 & $<0.0001$ \\
\hline Previous myocardial infarction & 464 & 29.7 & 695 & 23.9 & 877 & 27.4 & 188 & 31.3 & $<0.0001$ \\
\hline Serum creatinine $\geq 2 \mathrm{mg} / \mathrm{dl}$ & 46 & 2.9 & 105 & 3.6 & 120 & 3.8 & 22 & 3.7 & 0.548 \\
\hline Diabetes & 389 & 24.9 & 854 & 29.3 & 898 & 28.1 & 128 & 21.3 & $<0.0001$ \\
\hline Systolic PA pressure $>60 \mathrm{mmHg}$ & 13 & 0.8 & 30 & 1.0 & 38 & 1.2 & 2 & 0.3 & 0.231 \\
\hline Chronic pulmonary disease & 47 & 3.0 & 124 & 4.3 & 221 & 6.9 & 38 & 6.3 & $<0.0001$ \\
\hline NYHA III-IV & 144 & 9.2 & 267 & 9.2 & 363 & 11.3 & 81 & 13.5 & 0.001 \\
\hline Extracardiac arteriopathy & 229 & 14.6 & 697 & 23.9 & 1013 & 31.7 & 191 & 31.8 & $<0.0001$ \\
\hline Neurological dysfunction disease & 80 & 5.1 & 182 & 6.2 & 197 & 6.2 & 50 & 8.3 & 0.048 \\
\hline Previous cardiac surgery & 19 & 1.2 & 52 & 1.8 & 79 & 2.5 & 10 & 1.7 & 0.022 \\
\hline Single-vessel disease & 159 & 10.2 & 281 & 9.6 & 347 & 10.8 & 93 & 15.5 & 0.000 \\
\hline Double-vessel disease & 644 & 41.2 & 1141 & 39.2 & 1273 & 39.8 & 235 & 39.2 & 0.610 \\
\hline Triple-vessel disease & 761 & 48.7 & 1491 & 51.2 & 1580 & 49.4 & 272 & 45.3 & 0.048 \\
\hline Previous PCI & 280 & 17.9 & 427 & 14.7 & 463 & 14.5 & 73 & 12.2 & 0.002 \\
\hline Previous CABG & 14 & 0.9 & 40 & 1.4 & 65 & 2.0 & 7 & 1.2 & 0.014 \\
\hline Previous valve surgery & 2 & 0.1 & 2 & 0.1 & 6 & 0.2 & 1 & 0.2 & 0.642 \\
\hline Off-pump & 95 & 6.1 & 145 & 5.0 & 285 & 8.9 & 72 & 12.0 & $<0.0001$ \\
\hline LMCA disease & 31 & 2.0 & 80 & 2.7 & 104 & 3.3 & 28 & 4.7 & 0.005 \\
\hline Complete arterial grafts revascularization & 428 & 27.4 & 643 & 22.1 & 524 & 16.4 & 96 & 16.0 & $<0.0001$ \\
\hline
\end{tabular}

BMI: body mass index; LVEF: left ventricular ejection fraction; PA: pulmonary artery; NYHA: New York Heath Association; PCI: percutaneous coronary intervention; CABG: coronary artery bypass grafting; LMCA: left main coronary artery.

this was not statistically significant. They also reported a significantly more frequent history of previous PCI. However, older patients presented at surgery more often with higher EuroSCORE, due to the higher incidence of systemic comorbidities. We found that coronary revascularization was performed off-pump more frequently in patients over 60, whereas patients under 60 received more frequently onpump total arterial revascularization (Table 1 ).

At 30 days, mortality was $0.4 \%$ in patients under $60(6$ patients), $0.7 \%$ in those aged 60 to 69 years (20 patients), $2 \%$ in those aged 70 to 79 years (64 patients), and $3 \%$ in those aged 80 years or older (18 patients).

The mean follow-up of the overall cohort was $8.1 \pm 3.9$ years (median 8.26, min 1.2-max 14.4).

The unadjusted estimates of 5-year outcomes are summarized in Figures 3 and 4 . Younger patients reported significant better results than older patients in terms of overall mortality (Figure 3(a)), cardiac-related mortality (Figure 3(b)), AMI (Figure 4(a)), stroke (Figure 4(b)), and rehospitalization (Figure 5(a)). However, no difference between groups is detected in terms of need for repeat PCI (Figure 5(b)). Logrank test $p$ values were significant for all outcomes considered except repeat PCI, confirming the heterogeneity of outcome curves between age groups.

As a second step we performed 1:1 propensity score matching for patients under 60 and patients over 60 , in order to compare the outcomes of both groups with similar baseline and operative characteristics, except age. This matching yielded a cohort of 3128 patients, 1564 for each group (Table 2).

In this PS matched population, patients under 60 reported significantly lower overall mortality (at 5 years, 5.1\% versus 9.3\%; KM log-rank test $p<0.0001$ ) (Figure 6(a)), cardiac-related mortality (at 5 years, $2.1 \%$ versus $6.9 \%$; KM log-rank test $p<0.0001$ ) (Figure 6(b)), incidence of AMI (at 5 years, 3\% versus 6.5\%; KM log-rank test $p=0.01$ ) (Figure $7(\mathrm{a})$ ), and stroke rates (at 5 years, $2.1 \%$ versus $6.1 \%$; KM $\log$-rank test $p<0.0001$ ) (Figure $7(\mathrm{~b})$ ). No differences were reported between groups in terms of need for rehospitalization (at 5 years, 19.9\% versus 22.8\%; KM log-rank test $p=0.38$ ) (Figure 8(a)). Patients under 60 required repeated revascularization more frequently than older patients (at 5 years, $7.2 \%$ versus $5.9 \%$; $\mathrm{KM}$ log-rank test $p=0.05$ ) (Figure 8(b)). 


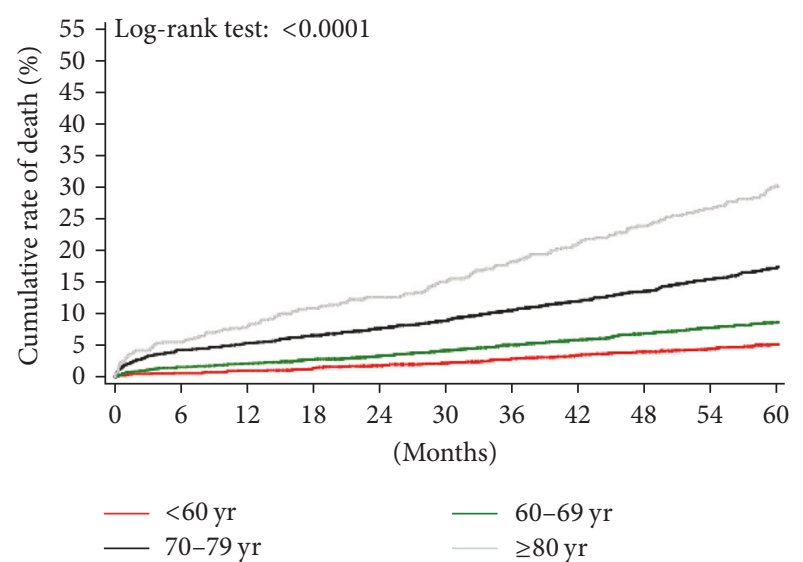

$\%$ patients at risk

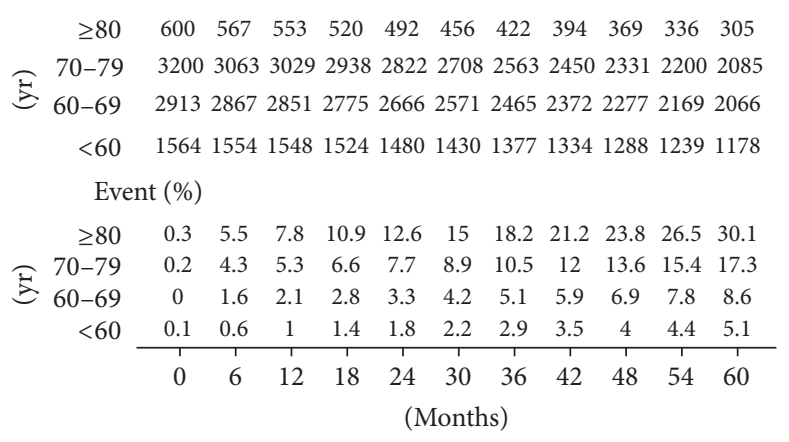

(a)

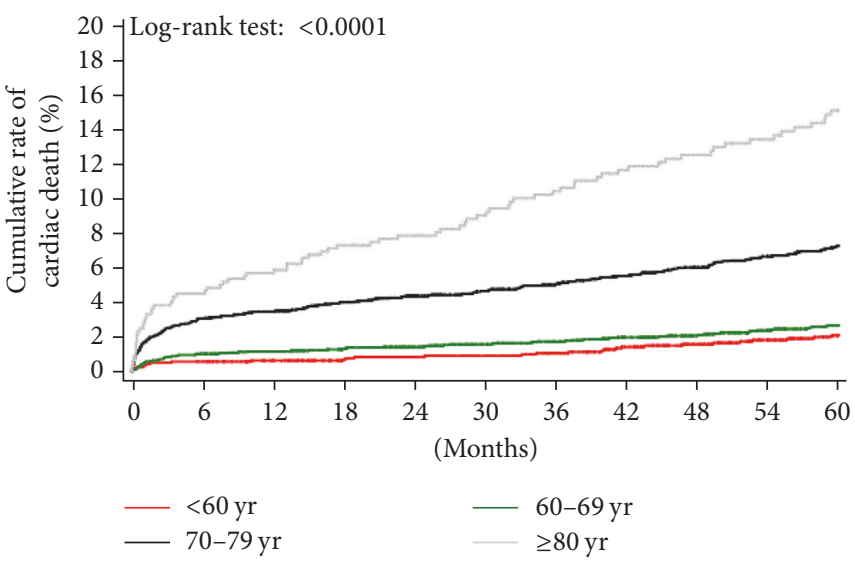

$\%$ patients at risk

$\begin{array}{llllllllllll}\geq 80 & 600 & 570 & 541 & 512 & 484 & 459 & 445 & 420 & 398 & 373 & 347\end{array}$

70-79 32003094298928852784269326002505241423022193

E $60-69 \quad 29132876278026852605252124332342225921652076$

$<60 \quad 15641553151814711426138713441298125311931139$

$$
\text { Event (\%) }
$$

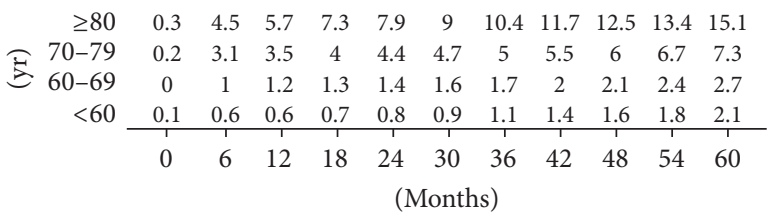

(b)

Figure 3: Kaplan-Meier estimates of late outcome in overall study population. (a) cumulative all-cause death; (b) cardiac-related death.

Table 3 reports multivariate analysis with significant independent predictors of mortality at 5 years.

\section{Discussion}

Recent studies conducted in Western Countries have found that the incidence of $\mathrm{CAD}$ has declined in the general population over the last few decades $[15,16]$, probably due to better prevention of cardiovascular risk. On the other hand, the incidence of CAD, including acute coronary artery syndromes, among young to middle-aged adults has been shown to have increased [15-17]. Previous studies of CAD in young adults have mostly been single-center analyses $[3,6]$, and few have been designed with the aim of studying young patients undergoing coronary revascularization $[3,5,7,18-$ 20].

The aim of this retrospective multicenter registry study was to investigate age-dependent trends in mortality, longterm survival, and comorbidity over time in a large population of patients undergoing isolated CABG.

The main findings of this study are as follows. Patients under 60 years of age who underwent CABG had lower long-term mortality and morbidity than older patients. Of particular interest is that at 5 years the $<60$ group reported unadjusted significantly lower cumulative rates of all-cause death, cardiac-related death, AMI, stroke, and rehospitalization. No difference between groups was detected in terms of need for repeat PCI.
In the matched population, patients under 60 reported significantly lower overall mortality, lower cardiac-related mortality, lower incidence of AMI, and a lower stroke rate. However, no differences were reported between groups in terms of need for rehospitalization. Finally, patients under 60 required repeated revascularization more frequently than older patients.

It is well known that cardiovascular risk factors vary with regard to their impact on age of presentation with CAD. Our study confirms that patients younger than 60 have a different clinical pattern of presentation of CAD in comparison with elderly patients. Particularly, male gender, obesity, the history of previous myocardial infarction, the presence of depressed LVEF, and a history of previous PCI have been found to be highly prevalent among patients $<60$, confirming the results of a recent study by Moussa et al. [14].

Obesity has already been recognized as an independent risk factor for CAD [21], and recently a close association between severity of obesity, measured by BMI $>30 \mathrm{~kg} / \mathrm{m}^{2}$, and a progressive reduction in the mean age of patients with symptomatic CAD has been demonstrated [22]. In particular, abdominal obesity has been found to be closely associated with the risk of myocardial infarction [23] and this is observed more often in men [24]. On the basis of these observations and our results, it is reasonable to postulate that the risk of CAD due to obesity may be higher in men than women. The higher proportion of males in the young 
TABLE 2: Baseline characteristics and operative data of patients as adjusted by multiple propensity score.

\begin{tabular}{|c|c|c|c|c|c|c|}
\hline \multirow{2}{*}{$\begin{array}{l}\text { Patients' characteristics } \\
\text { Female }\end{array}$} & \multicolumn{2}{|c|}{$<60(N=1564)$} & \multicolumn{2}{|c|}{$\geq 60(N=1564)$} & \multirow{2}{*}{$\begin{array}{c}p \text { value } \\
0.6775\end{array}$} & \multirow{2}{*}{$\begin{array}{c}\text { Standardized differences } \\
-0.015\end{array}$} \\
\hline & 154 & 9.8 & 161 & 10.3 & & \\
\hline BMI $\geq 30$ : obesity & 402 & 25.7 & 409 & 26.2 & 0.7752 & -0.01 \\
\hline Logistic EUROscore > 15\% & 3 & 0.2 & 0 & 0.0 & 0.0831 & 0.062 \\
\hline Critical preoperative state & 21 & 1.3 & 12 & 0.8 & 0.1152 & 0.056 \\
\hline Unstable angina & 101 & 6.5 & 76 & 4.9 & 0.053 & 0.069 \\
\hline Ejec. fraction $\leq 30 \%$ & 44 & 2.8 & 35 & 2.2 & 0.3051 & 0.037 \\
\hline Ejec. fraction $30 \%-60 \%$ & 370 & 23.7 & 375 & 24.0 & 0.8338 & -0.008 \\
\hline Previous myocardial infarction & 464 & 29.7 & 470 & 30.1 & 0.8147 & -0.008 \\
\hline Serum creatinine $\geq 2 \mathrm{mg} / \mathrm{dl}$ & 46 & 2.9 & 15 & 1.0 & $<0.0001$ & 0.144 \\
\hline Diabetes & 389 & 24.9 & 390 & 24.9 & 0.967 & -0.001 \\
\hline Systolic PA pressure $>60 \mathrm{mmHg}$ & 13 & 0.8 & 4 & 0.3 & 0.0286 & 0.078 \\
\hline Chronic pulmonary disease & 47 & 3.0 & 100 & 6.4 & $<0.0001$ & -0.161 \\
\hline NYHA III-IV & 144 & 9.2 & 148 & 9.5 & 0.8058 & -0.009 \\
\hline Extracardiac arteriopathy & 229 & 14.6 & 273 & 17.5 & 0.0321 & -0.077 \\
\hline Neurological dysfunction disease & 80 & 5.1 & 391 & 25.0 & $<0.0001$ & -0.579 \\
\hline Previous cardiac surgery & 19 & 1.2 & 8 & 0.5 & 0.0335 & 0.076 \\
\hline Single-vessel disease & 159 & 10.2 & 94 & 6.0 & $<0.0001$ & 0.153 \\
\hline Double-vessel disease & 644 & 41.2 & 790 & 50.5 & $<0.0001$ & -0.188 \\
\hline Triple-vessel disease & 761 & 48.7 & 680 & 43.5 & 0.0037 & 0.104 \\
\hline Previous PCI & 280 & 17.9 & 277 & 17.7 & 0.8885 & 0.005 \\
\hline Previous CABG & 14 & 0.9 & 5 & 0.3 & 0.0384 & 0.074 \\
\hline Previous valve intervention & 2 & 0.1 & 1 & 0.1 & 0.5635 & 0.021 \\
\hline Off-pump & 95 & 6.1 & 87 & 5.6 & 0.5412 & 0.022 \\
\hline LMCA disease & 31 & 2.0 & 27 & 1.7 & 0.596 & 0.019 \\
\hline Total arterial revascularization & 428 & 27.4 & 430 & 27.5 & 0.9361 & -0.003 \\
\hline
\end{tabular}

BMI: body mass index; LVEF: left ventricular ejection fraction; PA: pulmonary artery; NYHA: New York Heath Association; PCI: percutaneous coronary intervention; CABG: coronary artery bypass grafting; LMCA: left main coronary artery.

TABLE 3: Predictors for 5-years mortality risk (Cox proportional hazards model).

\begin{tabular}{lccc}
\hline Parameter & Hazard ratio & $95 \%$ hazard ratio confidence interval & $p$ \\
\hline Age $<60$ yrs & 0.3 & 0.2 & 0.5 \\
LVEF $\leq 30 \%$ & 2.2 & 1.4 & 3.6 \\
Previous myocardial infarction & 1.3 & 1.1 & 1.6 \\
Serum creatinine $\geq 2 \mathrm{mg} / \mathrm{dl}$ & 2.2 & 1.5 & 0.002 \\
Diabetes & 1.5 & 1.3 & 0.004 \\
Chronic pulmonary disease & 1.8 & 1.3 & 1.8 \\
NYHA III-IV & 1.5 & 1.2 & 2.5 \\
Extracardiac arteriopathy & 1.7 & 1.4 & 0.0001 \\
Previous CABG & 2.5 & 1.3 & 0.0002 \\
Off-pump & 2.3 & 1.6 & 0.001 \\
LMCA disease & 2.3 & 1.2 & 2.1 \\
\hline
\end{tabular}

LVEF: left ventricular ejection fraction; NYHA: New York Heath Association; CABG: coronary artery bypass grafting; LMCA: left main coronary artery.

population undergoing CABG in our study is not surprising, given that it is widely recognized that CAD occurs 7 to 10 years earlier in men than women $[25,26]$.

On the other hand, systemic comorbidities usually associated with severe $\mathrm{CAD}$, such as chronic pulmonary disease, diabetes, stroke, and extracardiac arteriopathy in our study, proved to be less frequent in patients aged $<60$. This is consistent with the previous international literature [27, 28] and may be explained by the fact that the onset of diabetes mellitus and systemic hypertension usually occurs later in life, and their effect on the pathogenesis of CAD may require several years or decades to become clinically evident. The purpose of this study was not to primarily investigate the risk factors determining premature coronary artery disease, but 


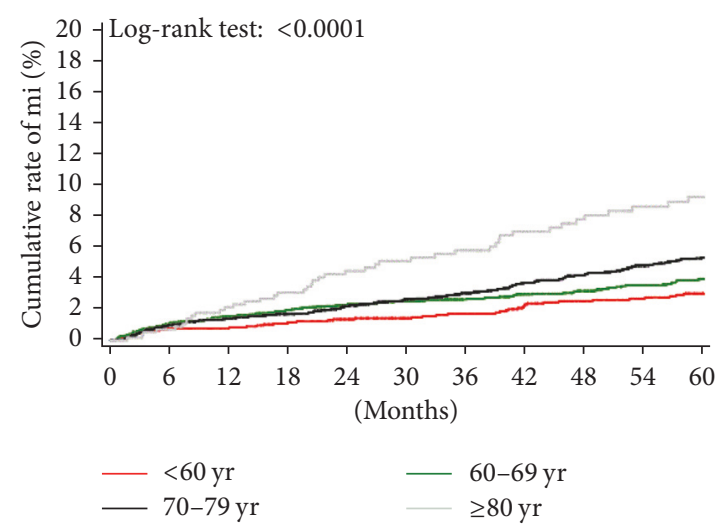

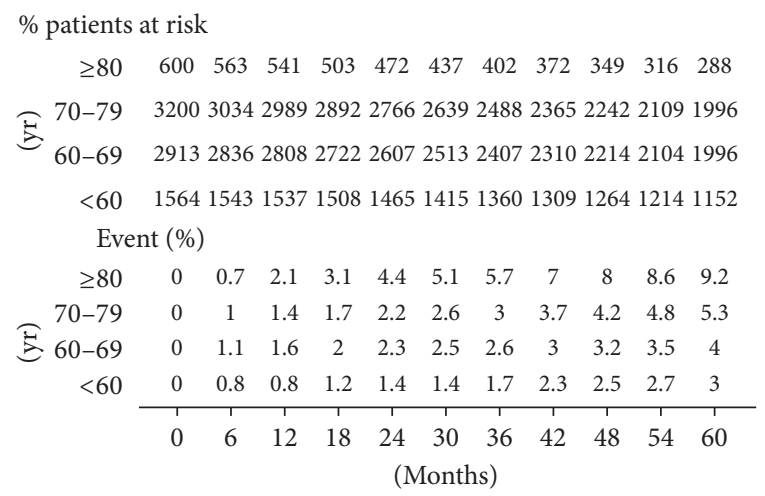

(a)

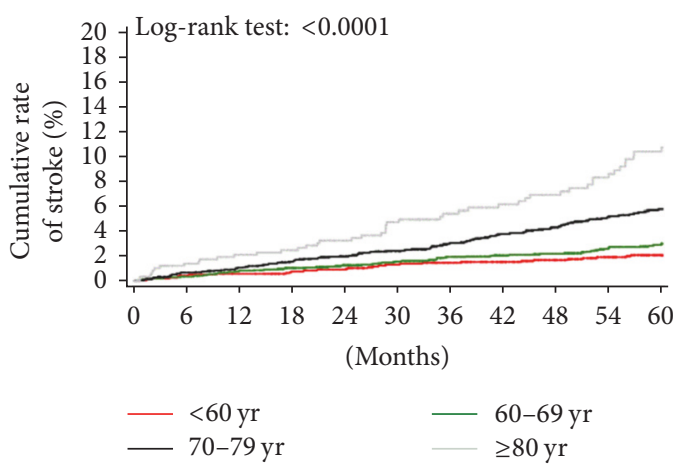

$\%$ patients at risk

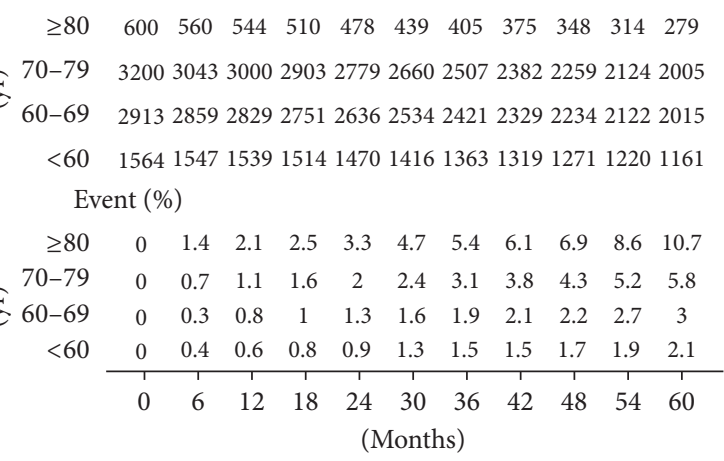

(b)

Figure 4: Kaplan-Meier estimates of late outcome in overall study population. (a) Myocardial infarction; (b) stroke.

our findings clearly confirm that the pathogenesis of coronary artery disease remains complex and that both genetic and environmental factors contribute to the early onset of coronary artery disease.

Long-term mortality was considerably lower in patients < 60 years than in patients $>60$, and this result is consistent with mortality rates reported in previous studies of young patients undergoing CABG [7, 14, 17, 29]. The long-term efficacy of CABG in patients $<60$ is further confirmed by a significantly higher freedom from AMI and stroke in comparison to older patients. Systemic comorbidities usually associated with severe $\mathrm{CAD}$, such as chronic pulmonary disease, diabetes, and extracardiac arteriopathy in our study, were found to be less frequent in patients aged $<60$, and this contributes to the lower postoperative and long-term morbidity reported in this subgroup of patients. In fact, in our study multivariate analysis confirmed that all classic clinical cardiac conditions and systemic comorbidities (severely depressed left ventricular function, history of previous myocardial infarction, NYHA classes III-IV, chronic renal failure, diabetes, chronic pulmonary disease, extra-cardiac arteriopathy, previous CABG, revascularization with off-pump technique, and left main coronary disease) are independent risk factors for mortality at 5 years.

Only $27.4 \%$ of patients $<60$ years received complete arterial graft revascularization in our study. A large body of scientific evidence demonstrates survival and repeat revascularization benefits of bilateral internal mammary artery grafting $[30,31]$. It is however the case that results of a recent ART Trial did not confirm significantly better mortality and morbidity rates at 5 years in patients who received bilateral mammary artery grafts in comparison to those, with similar mean age, who had traditional revascularization with single mammary artery grafts and saphenous vein grafts [32]. Nevertheless, in our study patients under 60 received total arterial revascularization more frequently than other age subgroups, and this may have potentially contributed to better long-term outcomes reported in this subgroup of patients. However, it appears that the clearest survival benefit of bilateral internal mammary artery grafting occurs after 5 years of follow-up and continues to increase 20 years after CABG [31].

The significantly higher rate of total arterial revascularization, which does not usually require intraoperative aortic manipulation, and the lower rate of extracardiac arteriopathy reported in younger patient subgroups may also partly explain the significantly better stroke rates in patients under 60 .

Despite the favorable outcomes reported in patients $<60$ undergoing $\mathrm{CABG}$, this study demonstrated that repeated revascularization occurred similarly in all the subgroups of unadjusted population and was more frequent in patients < 60 after PS matching. Possible explanations involve the lower amount of daily activity of older patients, so that they are less exposed to the potential recurrence of angina and the reluctance of older patients to undergo repeat revascularization 

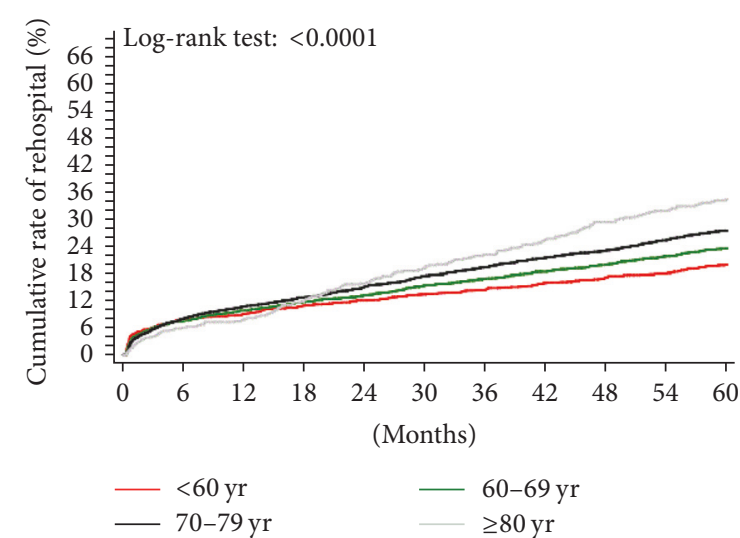

$\%$ patients at risk

$\begin{array}{llllllllllll}\geq 80 & 600 & 535 & 517 & 471 & 435 & 395 & 363 & 329 & 296 & 270 & 244\end{array}$

F 70-79 32002830272226052451230821592029191817851667

60-69 29132658258324712347221320961996189417671654

$<60 \quad 1564143114071362131312531211115811071059992$ Event (\%)

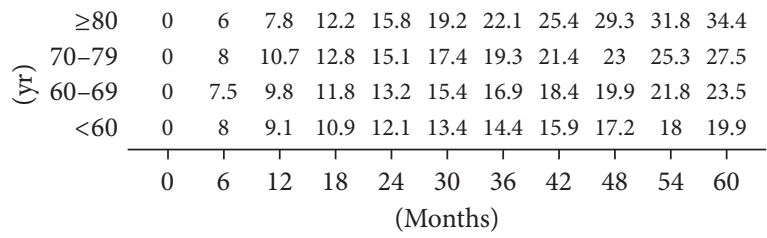

(a)

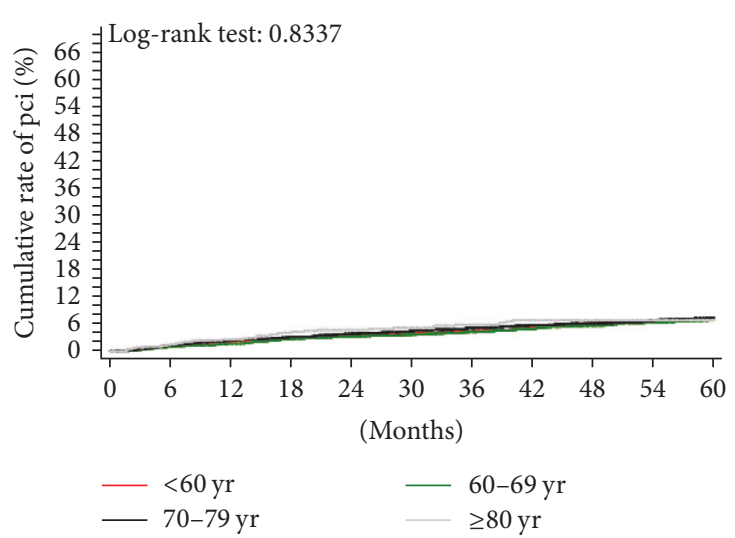

$\%$ patients at risk

$\begin{array}{llllllllllll}\geq 80 & 600 & 558 & 539 & 499 & 471 & 434 & 400 & 369 & 345 & 317 & 287\end{array}$

I 70-79 32003025296028512719259524422320220020661950

S 60-69 29132838280427002580248023642262216120471938

$<60 \quad 15641534151514821432 \quad 1378 \quad 1326 \quad 127112251172 \quad 1110$

Event (\%)

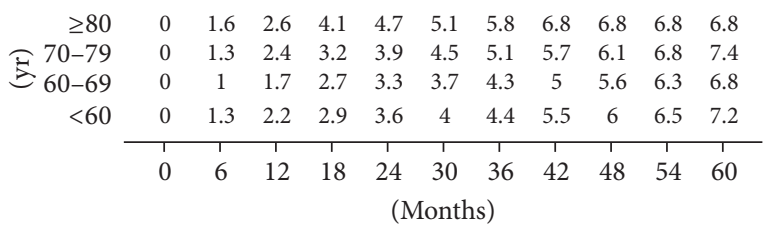

(b)

FIGURE 5: Kaplan-Meier estimates of late outcome in overall study population. (a) Repeat hospitalization; (b) repeat revascularization with PCI.

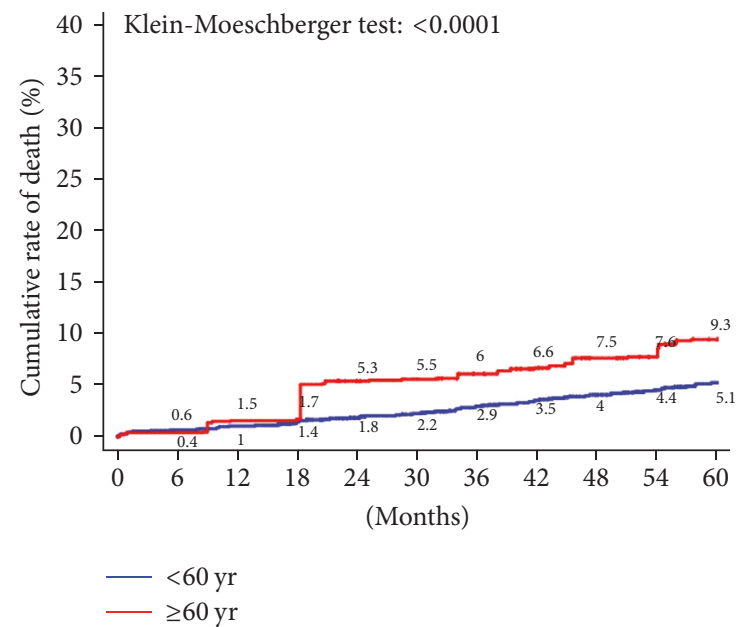

Patients at risk

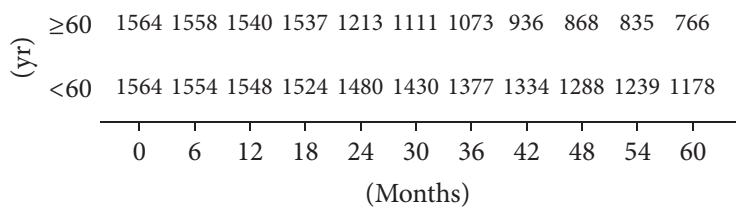

(a)

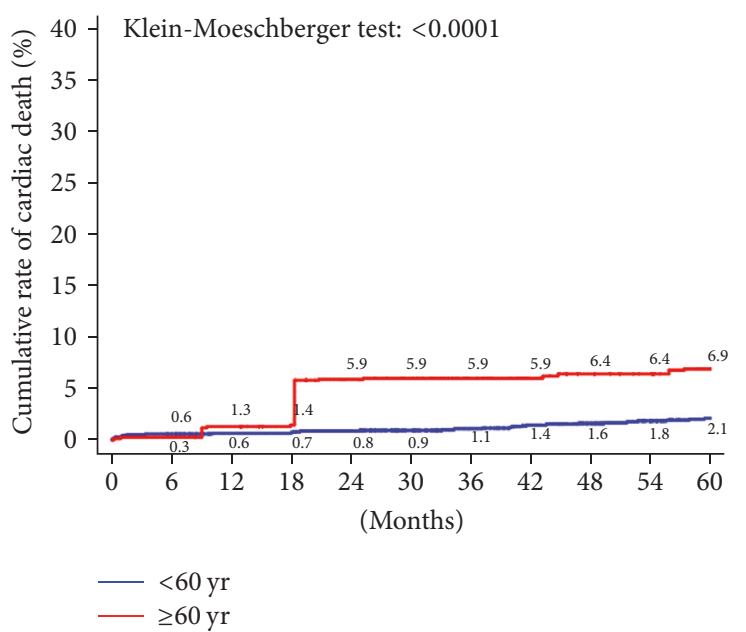

Patients at risk

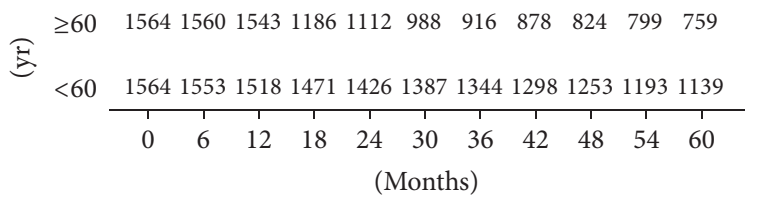

(b)

FIGURE 6: Kaplan-Meier estimates of late outcome in propensity score-matched pairs. (a) Cumulative all-cause death; (b) cardiac-related death. 


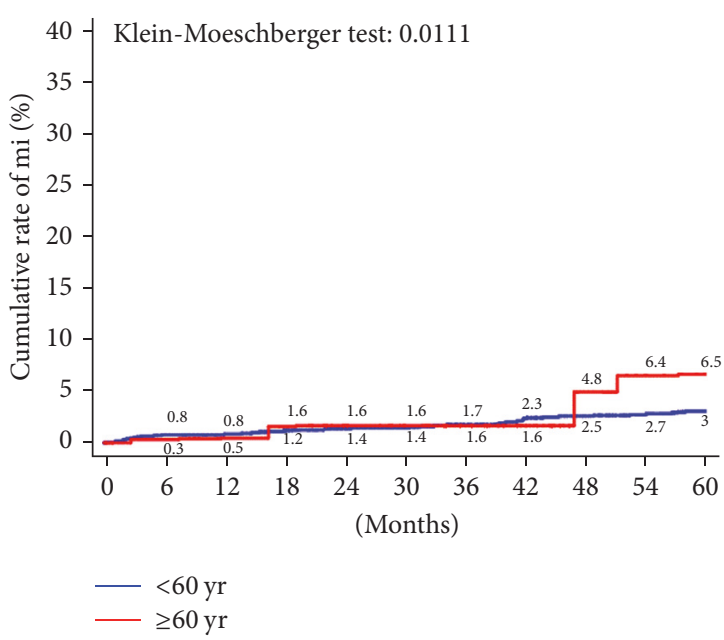

Patients at risk

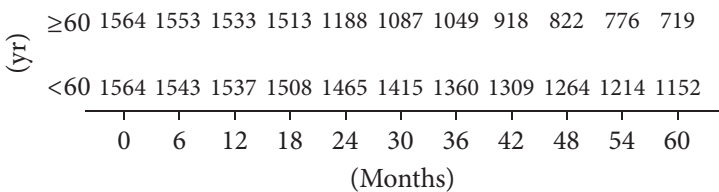

(a)

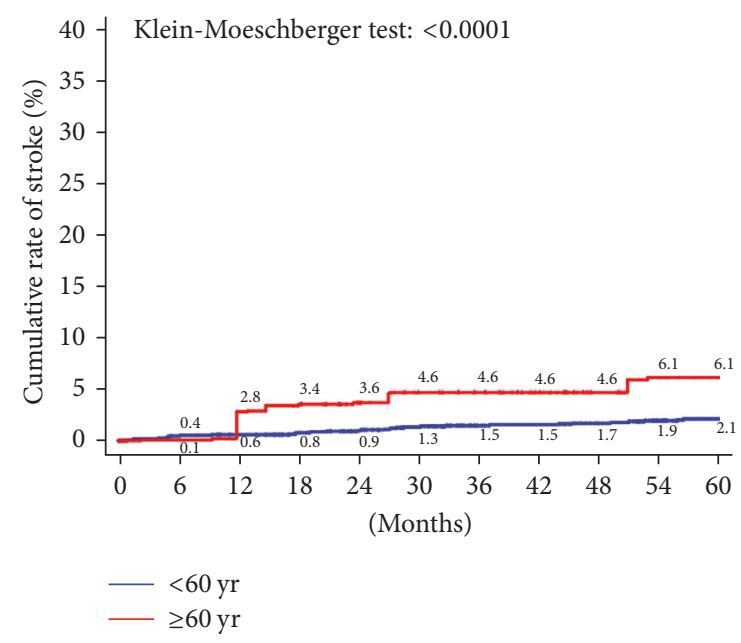

Patients at risk

$\widehat{E}$

$<6015641547153915141470141613631319127112201161$

$\begin{array}{lllllllllll}0 & 6 & 12 & 18 & 24 & 30 & 36 & 42 & 48 & 54 & 60\end{array}$

(Months)

(b)

FIGURE 7: Kaplan-Meier estimates of late outcome in propensity score-matched pairs. (a) myocardial infarction; (b) stroke.

procedures. On the other hand, younger patients are generally followed more closely by the cardiologist, with more frequent check-ups, and they may be given more aggressive medical therapy, but it is also reasonable to admit that they have a lower threshold for performing repeat revascularization in the case of mild angina symptoms.

The strength of this study is that it is a regional multicenter study including almost all consecutive patients undergoing isolated CABG surgery in Emilia-Romagna region (Italy) in a 12 -year period. The study population is large, and there is a long follow-up period (mean follow-up 8.1 years).

There are, however, several limitations inherent in the observational design of the study which need to be acknowledged.

We had no information about the patients not resident in the ER region and excluded from the follow-up analysis because of incomplete information on their clinical status after discharge from hospital. Although we tried to rigorously adjust selection bias using propensity score-based analysis, unmeasured confounders and hidden biases may have affected our results. The choice of strategy to perform CABG (off- versus on-pump CABG; total arterial revascularization versus traditional revascularization) was strongly affected by the surgeons' preferences, as well as by several other important baseline demographic and clinical profiles of the patients enrolled in the entire study cohort. The propensity score analysis could thus be slightly imperfect, and we were unable to completely adjust for hidden selection biases. Moreover, only clinical outcomes were assessed in this study, and graft patency was not assessed during the follow-up. Finally, we have no information on vessels (grafts or native coronary arteries) requiring repeat revascularization in the follow-up.
However, it should be recognized that some end-points as cardiac-related death and nSTEMI are not so easy to define and to collect in the follow-up of such a retrospective study, constituting a limitation in the evaluation of adverse outcomes in this patient population.

In conclusion, patients $<60$ years of age who underwent CABG had a lower risk of adverse outcomes than older patients. Of particular interest is that at 5 years the $<60$ group reported unadjusted and adjusted significantly lower cumulative rates of all-cause death in comparison to other groups. Patients $<60$ have a different clinical pattern of presentation of CAD in comparison with elderly patients. In particular, male gender, obesity, the history of previous myocardial infarction, the presence of depressed left ventricular function, and the history of previous PCI were found to be very prevalent among patients $<60$. These issues require great attention in the design and improvement of preventive strategies for reducing the impact of specific cardiovascular risk factors for younger patients, such as diet, lifestyle, and weight control.

Further studies with longer follow-up periods need to be conducted with the aim of studying the efficacy and durability of myocardial revascularization in younger patients who require $\mathrm{CABG}$.

\section{Conflicts of Interest}

The authors declare that they have no conflicts of interest.

\section{Acknowledgments}

List of RERIC (Registro dell'Emilia Romagna degli Interventi Cardiochirurgici) Investigators is as follows: G. A. Contini 


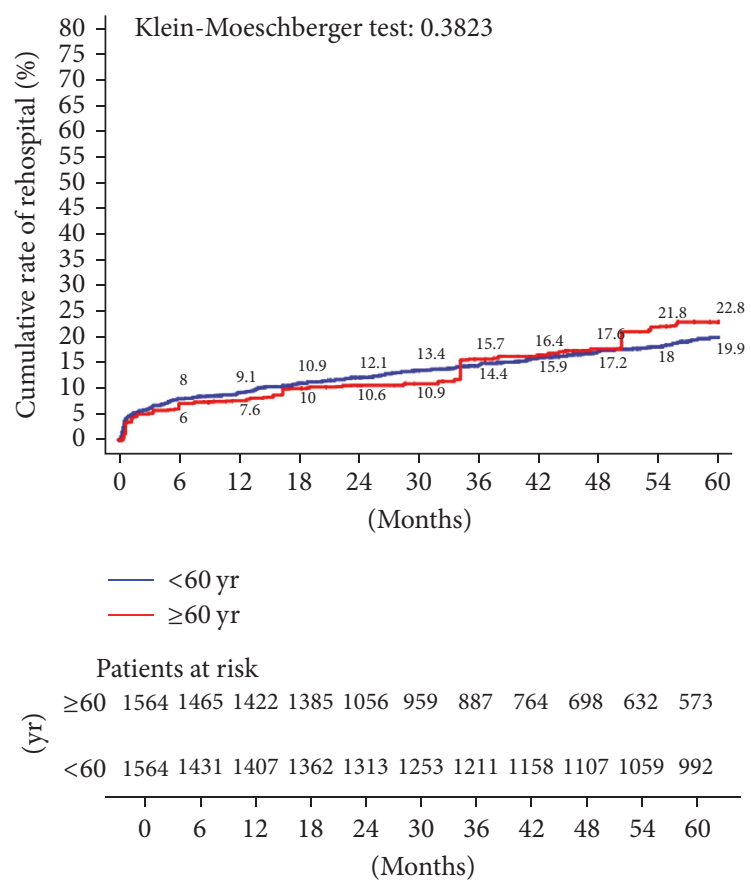

(a)

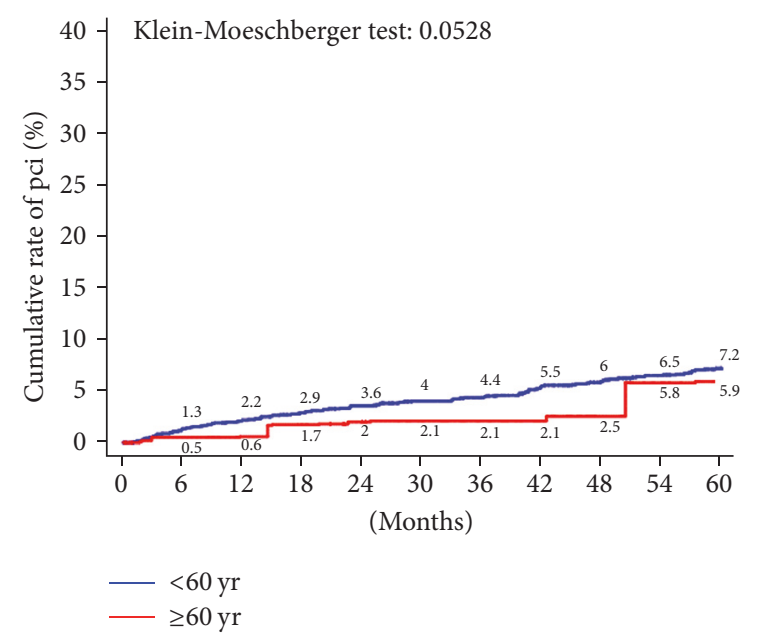

Patients at risk

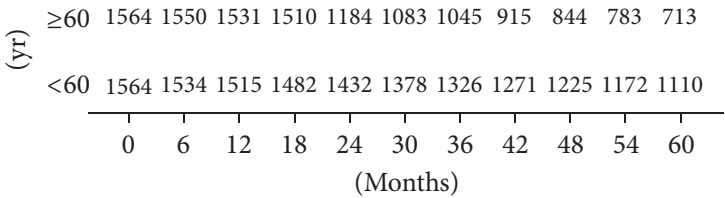

(b)

FIGURE 8: Kaplan-Meier estimates of late outcome in propensity score-matched pairs. (a) Repeat hospitalization; (b) repeat revascularization with PCI.

(Parma), D. Gabbieri (Modena), D. Pacini (Bologna), F. Pigini (Bologna), and C. Zussa (Lugo-Ravenna). The authors thank Lois Clegg, English Language Teacher, University of Parma, for her assistance in the revision of the manuscript.

\section{References}

[1] L. W. Klein and S. Nathan, "Coronary artery disease in young adults," Journal of the American College of Cardiology, vol. 41, no. 4, pp. 529-531, 2003.

[2] P. D’Errigo, F. Biancari, A. Maraschini, S. Rosato, G. Badoni, and F. Seccareccia, "Thirty-day mortality after coronary artery bypass surgery in patients aged $<50$ years: Results of a multicenter study and meta-analysis of the literature," Journal of Cardiac Surgery, vol. 28, no. 3, pp. 207-211, 2013.

[3] F. J. Khawaja, C. S. Rihal, R. J. Lennon, D. R. Holmes, and A. Prasad, "Temporal trends (over 30 Years), clinical characteristics, outcomes, and gender in patients $\leq 50$ years of age having percutaneous coronary intervention," American Journal of Cardiology, vol. 107, no. 5, pp. 668-674, 2011.

[4] F. H. Zimmerman, A. Cameron, L. D. Fisher, and N. Grace, "Myocardial infarction in young adults: Angiographic characterization, risk factors and prognosis (coronary artery surgery study registry)," Journal of the American College of Cardiology, vol. 26, no. 3, pp. 654-661, 1995.

[5] D. Mukherjee, A. Hsu, D. J. Moliterno, A. M. Lincoff, M. Goormastic, and E. J. Topol, "Risk factors for premature coronary artery disease and determinants of adverse outcomes after revascularization in patients $\leq 40$ years old," American Journal of Cardiology, vol. 92, no. 12, pp. 1465-1467, 2003.

[6] J. H. Cole, J. I. Miller, L. S. Sperling, and W. S. Weintraub, "Longterm follow-up of coronary artery disease presenting in young adults," Journal of the American College of Cardiology, vol. 41, pp. 521-528, 2003.

[7] F. Biancari, T. Gudbjartsson, J. Heikkinen et al., "Comparison of 30-day and 5-year outcomes of percutaneous coronary intervention versus coronary artery bypass grafting in patients aged $=50$ years (the Coronary aRtery diseAse in younG adultS Study)," American Journal of Cardiology, vol. 114, pp. 198-205, 2014.

[8] K. J. Buth, R. A. Gainer, J.-F. Legare, and G. M. Hirsch, "The changing face of cardiac surgery: Practice patterns and outcomes 2001-2010," Canadian Journal of Cardiology, vol. 30, no. 2, pp. 224-230, 2014.

[9] P. Kurlansky, "Do octogenarians benefit from coronary artery bypass surgery: A question with a rapidly changing answer?" Current Opinion in Cardiology, vol. 27, no. 6, pp. 611-619, 2012.

[10] F. Vasques, E. Lucenteforte, R. Paone, A. Mugelli, and F. Biancari, "Outcome of patients aged $\geq 80$ years undergoing combined aortic valve replacement and coronary artery bypass grafting: A systematic review and meta-analysis of 40 studies," American Heart Journal, vol. 164, no. 3, pp. 410-el, 2012.

[11] F. Nicolini, A. Molardi, D. Verdichizzo et al., "Coronary artery surgery in octogenarians: Evolving strategies for the improvement in early and late results," Heart and Vessels, vol. 27, no. 6, pp. 559-567, 2012.

[12] F. Nicolini, G. A. Contini, D. Fortuna et al., "Coronary artery surgery versus percutaneous coronary intervention in octogenarians: Long-term results," The Annals of Thoracic Surgery, vol. 99, no. 2, pp. 567-574, 2015.

[13] D. Fortuna, F. Nicolini, P. Guastaroba et al., "Coronary artery bypass grafting vs percutaneous coronary intervention in a 'real-world' setting: A comparative effectiveness study based on 
propensity score-matched cohorts," European Journal of CardioThoracic Surgery, vol. 44, no. 1, Article ID ezt197, pp. e16-e24, 2013.

[14] I. D. Moussa, L. W. Klein, B. Shah et al., "Consideration of a new definition of clinically relevant myocardial infarction after coronary revascularization: An expert consensus document from the society for cardiovascular angiography and interventions (SCAI)," Journal of the American College of Cardiology, vol. 62, no. 17, pp. 1563-1570, 2013.

[15] E. S. Ford and S. Capewell, "Coronary heart disease mortality among young adults in the US from 1980 through 2002. Concealed leveling of mortality rates," Journal of the American College of Cardiology, vol. 50, pp. 2128-2132, 2002.

[16] L. J. Nedkoff, T. G. Briffa, D. B. Preen et al., "Age- and sexspecific trends in the incidence of hospitalized acute coronary syndromes in western Australia," Circulation: Cardiovascular Quality and Outcomes, vol. 4, no. 5, pp. 557-564, 2011.

[17] S. Agarwal, K. Sud, B. Thakkar, V. Menon, W. A. Jaber, and S. R. Kapadia, "Changing Trends of Atherosclerotic Risk Factors Among Patients With Acute Myocardial Infarction and Acute Ischemic Stroke," American Journal of Cardiology, vol. 119, no. 10, pp. 1532-1541, 2017.

[18] M. Flather, J.-W. Rhee, D. B. Boothroyd et al., "The effect of age on outcomes of coronary artery bypass surgery compared with balloon angioplasty or bare-metal stent implantation among patients with multivessel coronary disease: A collaborative analysis of individual patient data from 10 randomized trials," Journal of the American College of Cardiology, vol. 60, no. 21, pp. 2150-2157, 2012.

[19] J. H. Lichtman, Y. Wang, S. B. Jones et al., "Age and sex differences in inhospital complication rates and mortality after percutaneous coronary intervention procedures: Evidence from the NCDR ${ }^{\circledR}, "$ American Heart Journal, vol. 167, no. 3, pp. 376-383, 2014.

[20] H. Kaneko, J. Yajima, Y. Oikawa et al., "Impact of aging on the clinical outcomes of Japanese patients with coronary artery disease after percutaneous coronary intervention," Heart and Vessels, vol. 29, no. 2, pp. 156-164, 2014.

[21] Y. Lu, K. Hajifathalian, M. Ezzati, M. Woodward, E. B. Rimm, and G. Danaei, "Metabolic mediators of the effects of body-mass index, overweight, and obesity on coronary heart disease and stroke: a pooled analysis of 97 prospective cohorts with 1.8 million participants," The Lancet, vol. 383, no. 9921, pp. 970-983, 2014.

[22] S. M. Atique, B. Shadbolt, P. Marley, and A. Farshid, "Association Between Body Mass Index and Age of Presentation With Symptomatic Coronary Artery Disease," Clinical Cardiology, vol. 39, no. 11, pp. 653-657, 2016.

[23] S. Yusuf, S. Hawken, and S. Ounpuu, "Erratum: Effect of potentially modifiable risk factors associated with myocardial infarction in 52 countries (the INTERHEART study): Casecontrol study (Lancet (2004) 364 (937-952))," The Lancet, vol. 364, no. 9450, p. 2020, 2004.

[24] R. See, S. M. Abdullah, D. K. McGuire et al., "The Association of Differing Measures of Overweight and Obesity With Prevalent Atherosclerosis. The Dallas Heart Study," Journal of the American College of Cardiology, vol. 50, no. 8, pp. 752-759, 2007.

[25] A. H. E. M. Maas and Y. E. A. Appelman, "Gender differences in coronary heart disease," Netherlands Heart Journal, vol. 18, no. 12, pp. 598-603, 2010.

[26] K. Matsis, A. Holley, A. Al-Sinan, P. Matsis, P. D. Larsen, and S. A. Harding, "Differing Clinical Characteristics Between Young and Older Patients Presenting with Myocardial Infarction," Heart, Lung and Circulation, vol. 26, no. 6, pp. 566-571, 2017.

[27] L. Choudhury and J. D. Marsh, "Myocardial infarction in young patients," American Journal of Medicine, vol. 107, no. 3, pp. 254261, 1999.

[28] R. Reibis, A. Treszl, K. Wegscheider, K. Bestehorn, B. Karmann, and H. Völler, "Disparity in risk factor pattern in premature versus late-onset coronary artery disease: A survey of 15,381 patients," Vascular Health and Risk Management, vol. 8, no. 1, pp. 473-481, 2012.

[29] M. Dalén, T. Ivert, M. J. Holzmann, and U. Sartipy, "Coronary artery bypass grafting in patients 50 years or younger: a Swedish nationwide cohort study," Circulation, vol. 131, no. 20, pp. 17481754, 2015.

[30] G. Yi, B. Shine, S. M. Rehman, D. G. Altman, and D. P. Taggart, "Effect of bilateral internal mammary artery grafts on long-term survival: A meta-analysis approach," Circulation, vol. 130, no. 7, pp. 539-545, 2014.

[31] B. W. Lytle, E. H. Blackstone, J. F. Sabik, P. Houghtaling, F. D. Loop, and D. M. Cosgrove, "The effect of bilateral internal thoracic artery grafting on survival during 20 postoperative years," The Annals of Thoracic Surgery, vol. 78, no. 6, pp. 20052014, 2004.

[32] D. P. Taggart, D. G. Altman, A. M. Gray et al., "Randomized trial of bilateral versus single internal-thoracic-artery grafts," The New England Journal of Medicine, vol. 375, no. 26, pp. 25402549, 2016. 


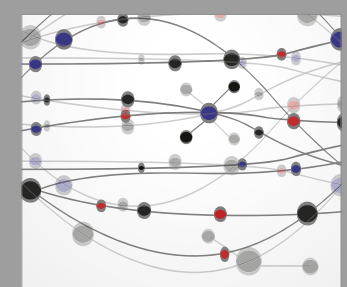

The Scientific World Journal
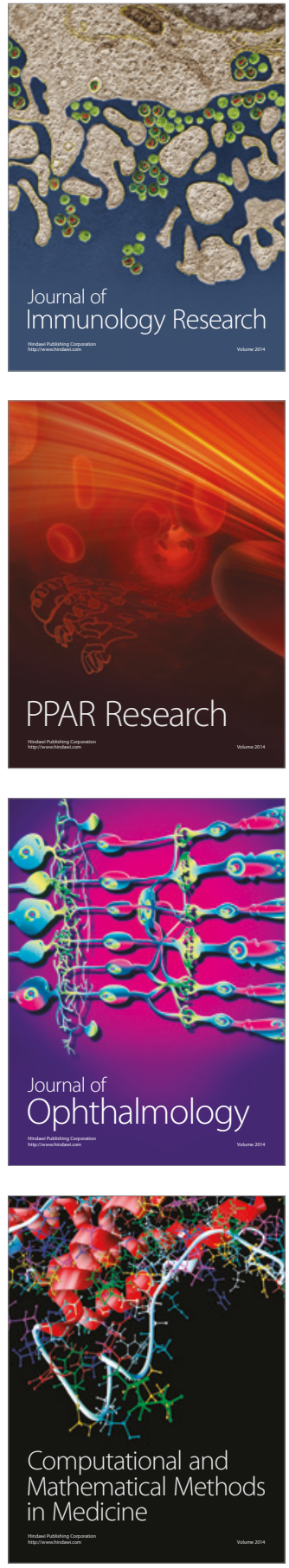

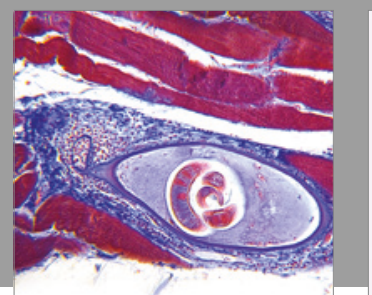

Gastroenterology Research and Practice
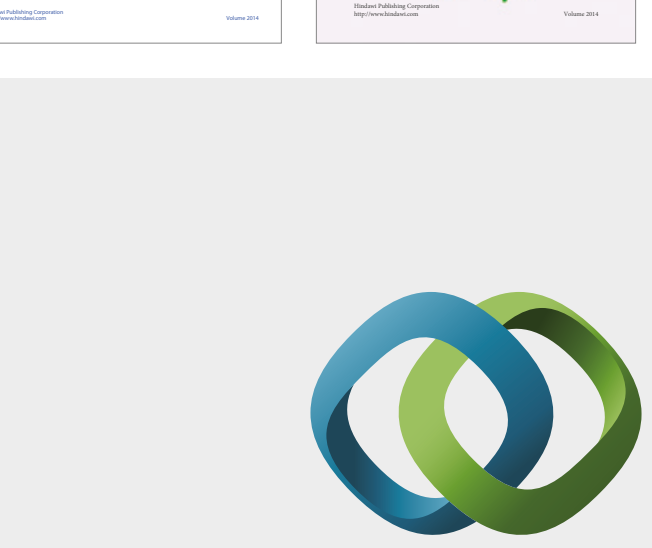

\section{Hindawi}

Submit your manuscripts at

https://www.hindawi.com
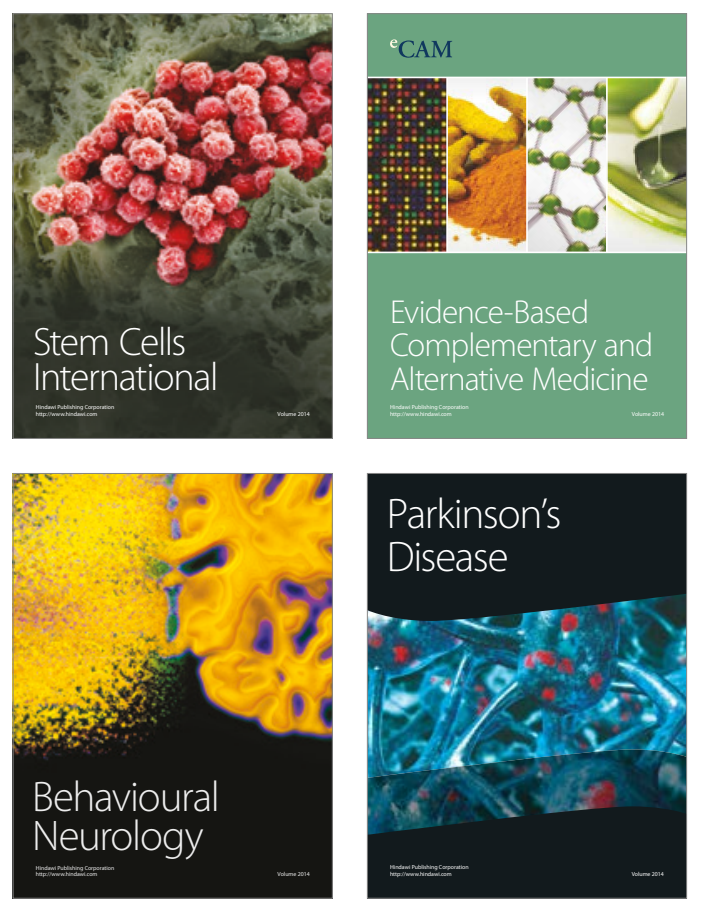
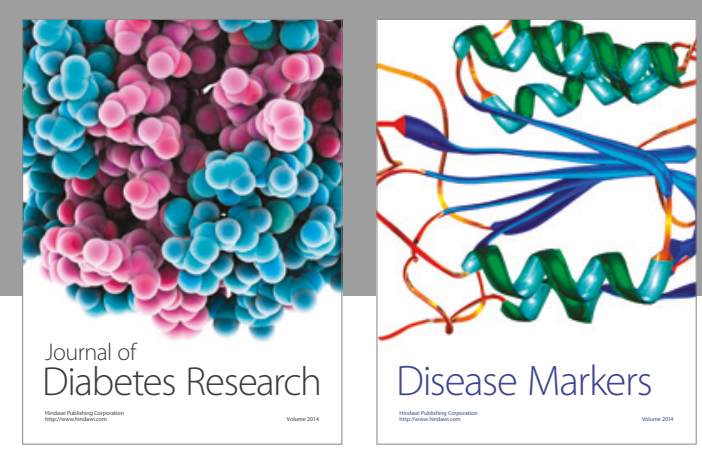

Disease Markers
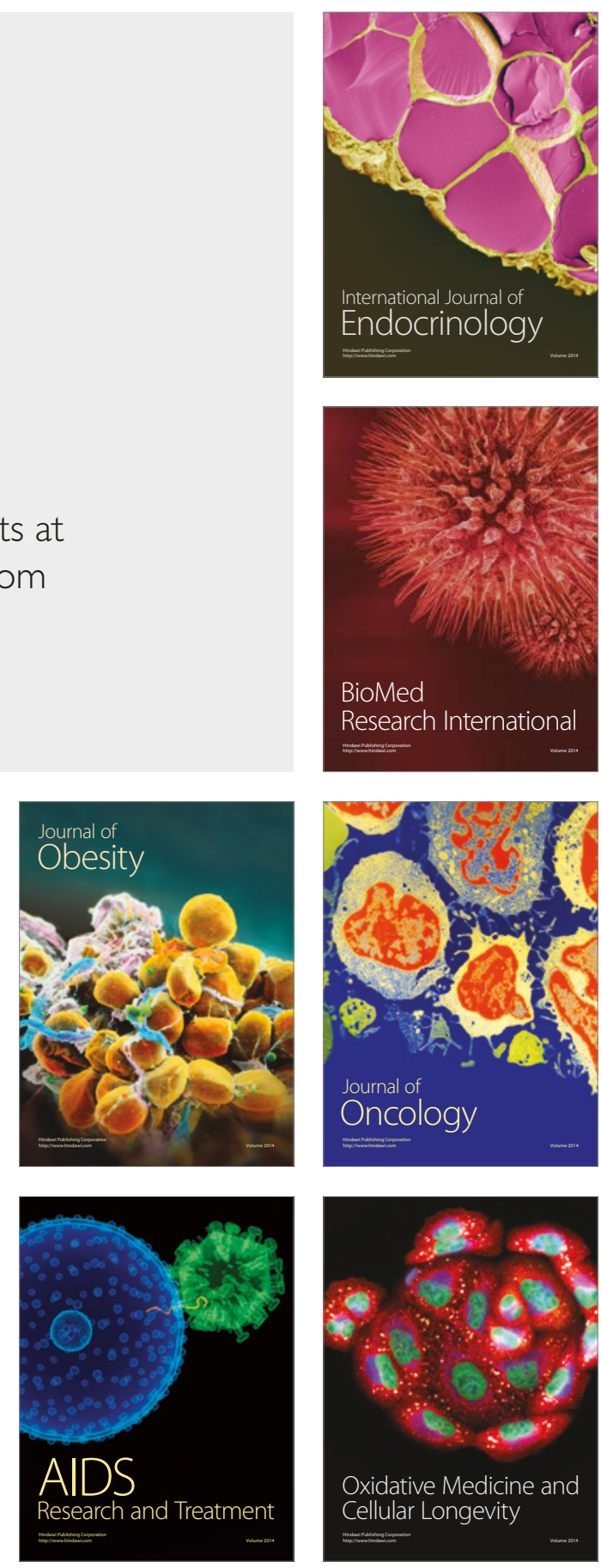